\title{
Treating Anxiety with a Beta Blocker - Antimuscarinic Combination: A Review of Compounded Atenolol - Scopolamine
}

\author{
Thomas P Dooley ${ }^{1 *}$, Ashley B Benjamin² and Ty Thomas ${ }^{3}$ \\ ${ }^{1}$ Trends in Pharma Development, LLC, 7100 Cabin Lane, Pinson, Alabama, USA \\ ${ }^{2}$ Department of Psychiatry, Ventura County, California, USA \\ ${ }^{3}$ National Center for Pain Management and Research, Vestavia Hills, Alabama, USA
}

*Corresponding author: Thomas P Dooley, Trends in Pharma Development, Alabama, USA, Tel: +2052226145; E-mail: tom@tomdooley.org

Received date: November 5, 2019; Accepted date: December 13, 2019; Published date: December 20, 2019

Citation: Dooley TP, Benjamin AB, and Thomas T (2019) Treating Anxiety with a Beta Blocker - Antimuscarinic Combination: A Review of Compounded Atenolol - Scopolamine. Clin Psychiatry Vol.5 No.3:63.

\section{Abstract}

A patented new class of anti-anxiety medications consists of a beta blocker plus an antimuscarinic agent to inhibit the sympathetic and parasympathetic symptoms of anxiety disorders, respectively. The $\operatorname{Pan}^{\circledR}$ medications are non-benzodiazepines to address the unmet medical need for fast-acting and effective anxiolytics, without using active ingredients known to produce dependence or addiction. The effects and side effects of compounded Atenolol - Scopolamine $\mathrm{HBr}$ were assessed in physiciansponsored studies in psychiatry and pain management settings. A total of 22 patients affected by Generalized Anxiety Disorder, Social Anxiety Disorder, Post-Traumatic Stress Disorder, and/or Panic Attack, or acute anxiety conditions (e.g., situational anxiety associated with a medical procedure or Substance Use Disorders) were treated prn with Atenolol $25 \mathrm{mg}$ - Scopolamine $\mathrm{HBr} 0.2$ $\mathrm{mg}$. Eighteen patients ( 82 percent) were responders to this combination drug approach. The patients perceived a calming effect in less than 30 minutes via oral mucosal delivery or less than 60 minutes by oral ingestion, and the perceived calming effect lasted up to 8 hours. The calming effect was further substantiated using a 0-10 point subjective anxiety score and the "State" anxiety symptom severity was assessed using the Beck Anxiety Instrument (BAI-S). Heart rate and blood pressure were reduced at 1 hour and 4-5 hours, consistent with the effects of a beta blocker.

Keywords: Anxiety; Anxiolytic; Atenolol; Scopolamine; Beta blocker; Antimuscarinic

\section{Introduction}

Anxiety disorders are common and serious conditions with huge public health impact [1]. The lifetime prevalence of Social Anxiety Disorder is $12.1 \%$, Panic Disorder is $4.7 \%$, and Generalized Anxiety Disorder (GAD) is $5.7 \%$ of the adult population in the United States [2]. The US population in 2017 is 325.4 million, of which $22.8 \%$ are under 18 years of age. Thus, there are 251.2 million adults. The 12-month prevalence of any anxiety disorder is $18.1 \%$. Therefore, an estimated 45.5 million adults are affected by anxiety disorders in the US.

While medications such as the SSRI and SNRI antidepressants and the serotonin 1a receptor partial agonist, buspirone, are approved and widely used as long-term prophylactics for anxiety disorders, the treatment of acute anxiety per se remains a challenge. The benzodiazepines are widely used and remain the mainstay of short-term anxiety treatment. However, the benzodiazepine class of medications has serious drawbacks, the most important of which is the potential for dependency and abuse [3]. In fact, four benzodiazepines are in the top 20 most abused prescription drugs and it is the second most commonly abused class of medications after opioids. Benzodiazepines also pose significant risks of side effects such as memory impairment, addiction, sedation, and other adverse events such as falls or other accidents. Furthermore, in 2016 the US FDA issued a warning against the coincident use of benzodiazepines and opioids. Benzodiazepines are also contributors to the current "Opioid Crisis" in the USA that claimed the lives of approximately 72,000 persons in 2017 . With the exception of benzodiazepines there are few other options available for the "as needed" (prn) treatment of anxiety, except for the approved antihistamine, hydroxyzine, and "off-label" beta blockers that are often prescribed for performance anxiety.

\section{Literature Review}

Although oral benzodiazepines and SSRIs have been used as persistent daily medications for the prophylaxis of anxiety disorders, they are either inappropriate or less than ideal medications for symptomatic treatment. Zamorski and Albucher stated, "Benzodiazepines should not be used on an as-needed basis for panic disorder. None of the oral benzodiazepines works quickly enough to affect any but the most prolonged panic attacks." [4]. Benzodiazepines can lead to physical dependency and dosage escalation in many patients [5]. Although SSRIs are considered as the first choice medications for anxiety disorders, they routinely require a 
slow dose-escalation approach over weeks of time. Thus, the most common drugs for anxiety indications - SSRIs and benzodiazepines - are not appropriate or are less than ideal for prn therapy for symptomatic relief, in view of inappropriate pharmacokinetics or side effects, respectively.

There is an unmet medical need for fast-acting and effective treatments for the symptoms of anxiety without using dependent/addictive ingredients. The $\operatorname{Pan} X^{\circledast}$ combination drugs are expected to address this unmet medical need [6]. The active pharmaceutical ingredient (API) combinations of Pan $X^{\circledR}$ provide complementary pharmacologic benefits by interaction of the two APIs with two or more pharmacologic molecular targets [6]. The biochemical pathways of the betaadrenergic and muscarinic gene families are appropriate targets, as they are involved in the regulation of the symptoms of anxiety. Those molecular targets can be components of the central nervous system (CNS), the peripheral nervous system, and/or of target somatic systems (e.g., cardiovascular and gastrointestinal). Given that the multiplicity of anxiety symptoms are under the regulation of different neurologic and neuroendocrine pathways, it follows that pharmacologic antagonists affecting both the beta adrenergic (i.e., beta 1) and muscarinic (i.e., $\mathrm{M} 1$ and/or $\mathrm{M} 2$ ) receptors could suppress more symptoms than could be achievable by a single API alone.

A stimulus or "trigger" circumstance that precipitates anxiety results in cortisol and epinephrine release, which causes multiple physiological effects. Epinephrine is responsible for the "fight-or-flight response". Symptoms of acute anxiety include tachycardia, palpitations, hyperventilation, dyspnea, increased blood pressure, anxiousness, nervousness, fear, avoidance, ruminating thoughts, nausea, vomiting, tremor, and sweating. These symptoms are under the control of both the sympathetic and parasympathetic autonomic nervous system, which are affected by beta blockers and antimuscarinic agents, respectively.

Beta blockers can suppress tachycardia, palpitations, increased blood pressure, and tremor, all of which are important symptoms of acute anxiety. Beta antagonist medications are typically administered for the treatment of hypertension. However, many physicians are aware of off-label use of beta blockers (e.g., Atenolol or Propranolol) for performance anxiety related to public speaking or musical performances. As stated by Zamorski and Albucher, "Beta blockers, once widely touted as effective antipanic medications, have proven disappointing as monotherapy in subsequent placebo-controlled trials." [4]. In other words, beta blockers provide some limited benefits in treating a subset of the symptoms of acute anxiety.

Antiemetic medications acting as muscarinic receptor antagonists can suppress nausea, vomiting, motion sickness, sea sickness, morning sickness in pregnancy, and other CNS symptoms of anxiety such as anxiousness, fear, and avoidance. Scopolamine is among the most potent antimuscarinic agents, and has been extensively reported in the literature for the treatment of motion sickness.
Beta blockers and antimuscarinic agents are included in the PanX ${ }^{\circledR}$ combinations not only for pharmacologic targeting of the sympathetic beta- 1 and parasympathetic M2/M1 receptors, but also in view of historic safety. Both classes of drugs have been used in millions of patients over five decades and are known to be safe. Neither class produces dependence or addition or is classified as a Controlled Substance.

In addition, the high prevalence of use strongly suggests that both classes of medicines (beta blockers and antimuscarinic agents) have been coincidentally and/or concurrently used within the same patients as separate prescriptions and/or OTC drugs. In other words, patients routinely taking a daily oral prescription beta blocker for hypertension (or other cardiovascular condition) coincidentally administer a prescription or OTC antimuscarinic agent for motion sickness, nausea, or vomiting. Even with high coincident and/or concurrent use, there is no evidence in the population of producing side effects or drug-drug interactions that extend beyond those inherent within either of the monotherapies. Historic safety of the active ingredients within $\mathrm{PanX}^{\circledR}$ combinations is a significant advantage vis-à-vis benzodiazepines with known serious adverse effects (most notably dependence and potential of abuse).

\section{Atenolol}

Atenolol is a beta-1 selective adrenergic receptor antagonist to reduce cardiovascular symptoms (e.g., tachycardia, palpitations, and hypertension) and at least another symptom (i.e., tremor) resulting from the acute release of epinephrine into the circulation. Beta blockers interfere with receptor binding by catecholamines, epinephrine and norepinephrine. The principal catecholamine affecting cardiovascular symptoms is epinephrine. Atenolol does not manifest dependence, addiction, abuse, or sedation that are common side effects with other anxiolytic medications, such as benzodiazepines. Atenolol and other beta blockers are prescribed for the treatment of various cardiovascular indications (with FDA approval), most notably hypertension, arrhythmia, angina, and prophylaxis of migraines. Atenolol is available as an FDA-approved oral drug at 25-100 mg/dose [7].

Human clinical studies have provided evidence that Atenolol can display some benefit with regard to the symptoms of acute anxiety. For instance, Atenolol has been used to suppress performance anxiety when administered orally in advance [8]. It has also been shown to be beneficial in alcohol withdrawal and flight phobia [9-12]. In a placebo-controlled trial Atenolol was found to be moderately effective against Social Anxiety Disorder [13]. In another placebo-controlled trial the pretreatment of patients with Atenolol 2 hours prior to a medical procedure (nasal speculum insertion) reduced the procedurerelated increase in tachycardia and mean arterial pressure [14]. Atenolol is a beta-1 selective peripheral-acting agent, which should reduce the risk for asthmatic and COPD subjects relative to the non-selective beta blockers, such as Propranolol $[15,16]$.

In view of these clinical reports, off-label Atenolol can provide some symptomatic relief with regard to performance 
anxiety. However, beta blockers alone do not sufficiently address the aggregate symptoms of acute anxiety, and especially the psychic symptoms thereof. Thus, there is a need to couple a beta-blocker with another type of active ingredient to affect the CNS and other symptoms.

\section{Scopolamine}

Scopolamine (also known as Hyoscine) is commonly used for the treatment of motion sickness, nausea, and vomiting. The mechanism of action of this antiemetic drug is as an antagonist of muscarinic acetylcholine receptors. Scopolamine is sold by prescription in the USA as a transdermal patch (Transderm Scop ${ }^{\circledR}$ ) that contains $1.5 \mathrm{mg}$ for dosing over three days. However, scopolamine is available as an over-the-counter (OTC) oral drug in Europe, Canada, UK, and some other foreign markets. OTC drugs are considered to be very safe by regulatory agencies that have granted the approvals. In Australia it is an oral OTC product with a recommended adult dose of $0.3 \mathrm{mg}$ (or $0.6 \mathrm{mg}$ ), and a maximum daily dose of 1.2 $\mathrm{mg}$ [17]. The foreign OTC is available for use in children at half of this dose. Scopolamine can be absorbed orally or mucosally, for the treatment of nausea and motion sickness symptoms $[18,19]$. Scopolamine $\mathrm{HBr}$ has been delivered sublingually at $0.15 \mathrm{mg} /$ dose in adults [18], orally at $0.3-1.0 \mathrm{mg} /$ dose in adults $[17,19,20]$, and transdermally at $0.5 \mathrm{mg} /$ day for 3 days in adults for motion sickness.

Scopolamine was known over a century ago to produce a calming effect in psychiatric patients [21], although this pharmacologic property is not well known today among medical providers. There is also evidence that scopolamine can exhibit an antidepressant property after multiple days when administered either intravenously [22-24] or orally [20] to patients with major depressive disorder (MDD). Furthermore, a human genetic study of the muscarinic acetylcholine receptor $\mathrm{M} 2$ (CHRM2) gene has revealed an association between specific genetic polymorphisms and the risk of depression in major depressive syndrome [25]. Scopolamine is a high potency muscarinic receptor antagonist that likely affects anxiety/mood via the M2 and/or M1 receptors of the CNS [26]

Although safely used worldwide for decades in Rx or OTC products, at high doses Scopolamine $\mathrm{HBr}$ can produce sedation and impaired vision/dilated pupils. It is considered to be a sedative at $1.2+\mathrm{mg} /$ dose in adults. [Note this is $6 \times$ higher than the dose in the $\operatorname{Pan} X^{\circledR}$ anxiolytic combination drug summarized below].

\section{PanX ${ }^{\circledR}$ drug combinations}

The $\operatorname{Pan}^{\circledR}$ drugs may be delivered by oral or mucosal routes. Delivery by the mucosal (e.g., sublingual) route avoids first-pass metabolism by the liver. In general oral delivery of drugs results in a "slower and lower" clinical effect relative to mucosal delivery. When "time is of the essence" for treatment of acute anxiety symptoms, convenient mucosal delivery would be preferred. An orally-disintegrating tablet (ODT) is intended to primarily deliver the APIs rapidly via the oral mucosal route (sublingual and buccal). However, some portion of the APIs might be delivered by the gastric route.

Patients may use the product in anticipation of an anxietyprovoking stimulus, for instance when encountering a known trigger circumstance. A trigger circumstance could include public speaking, a public performance, flying, driving, or a crowd of people (e.g., agoraphobia). The patient who expects to encounter a trigger could then self-administer the medication in advance. The medication is administered as a symptomatic therapy to be taken at the time of an episode or in anticipation of an episode. The product is intended to be readily available in a purse or pocket for ease of use, and with an anticipated duration of anxiolytic effect in hours.

Combination therapies can be sold to patients in need of a treatment, albeit subject to regulatory oversight. Compounded medications including multiple active ingredients are unapproved prescription products, and are produced under the FDA (DQSA) 503A or 503B regulations. Combination drug products may also be approved for specific medical indications through the FDA's IND/NDA drug approval processes.

"The PanX ${ }^{\circledR}$ beta blocker" antimuscarinic combination drugs were anticipated to produce a self-perceived calming effect in most individuals (and/or observed by an objective observer). The symptoms due to the autonomic nervous system (sympathetic and parasympathetic) that might be alleviated include tachycardia, palpitations, elevated blood pressure, nausea, vomiting, anxiousness, fear, avoidance, ruminating thoughts, aberrant breathing, sweating, tremors, migraine, and headache. The treatment was designed to reduce the severity of most or all of these somatic and/or psychic symptoms associated with acute anxiety.

The combination drugs were expected to be well tolerated in patients. The possible side effects might include dryness of the mouth (most likely with ODTs), bradycardia (<60 bpm), hypotension ( $<90 / 60 \mathrm{~mm} \mathrm{Hg}$ ), dizziness or light headedness, mild sedation, and although unlikely, blurred vision (pupil dilation). Impairment of cognition was not anticipated.

\section{Physician-sponsored studies of atenolol - scopolamine}

The compounded pharmaceutical composition utilized in the reported clinical studies consists of a combination of Atenolol $25 \mathrm{mg}$ and Scopolamine Hydrobromide $0.20 \mathrm{mg}$, in an ODT. This compounded drug has been shown in multiple physician-sponsored clinical case series to have beneficial features as a prn anxiolytic treatment [27-30]. In addition, physician-sponsored open label studies have demonstrated effectiveness in psychiatry and in situational anxiety in patients undergoing a medical procedure [31,32].

The effects and side effects of the 22 patients treated with Atenolol - Scopolamine $\mathrm{HBr}$ are summarized in Table 1. The physician-sponsored studies with informed consent demonstrated the effectiveness, tolerability, and safety of compounded Atenolol $25 \mathrm{mg}$ - Scopolamine HBr $0.2 \mathrm{mg}$ : 
Open label study in psychiatry: Eight patients were diagnosed with various anxiety disorders and treated in an open label study of Atenolol $25 \mathrm{mg}$ - Scopolamine $0.2 \mathrm{mg}$ [32]. All 6 responders were diagnosed by a psychiatrist with Generalized Anxiety Disorder plus one or more additional comorbid diagnoses, such as Social Anxiety Disorder $(n=4)$, Major Depressive Disorder $(n=4)$, PTSD $(n=2)$, and Opioid and Benzodiazepine Use Disorder $(n=1)$. The "State" anxiety was assessed using the Beck Anxiety Inventory instrument (BAI-S) as the primary endpoint pre-treatment versus post- treatment. The mean BAI-S in all 8 patients reduced from 15.9 pre-treatment to 8.5 at 1 hour and 4.1 at 4 hours. This substantial reduction was evident in spite of these individuals being considered by the psychiatrist as difficult-to-treat psychiatric patients, and two were non-responders. In another assessment, the mean of the subjective anxiety level (0-10 point scale) in the six responders was 7.1 pre-treatment versus 2.4 post-treatment. Thus, both the BAI-S and subjective anxiety scores reduced substantially on $\mathrm{PanX}^{\circledR}$ Atenolol Scopolamine.

Table 1: Summary of Physician-Sponsored Studies of Compounded Atenolol - Scopolamine.

\begin{tabular}{|l|l|l|l|}
\hline Medical Speciality & \# Responders & Conditions & Evidences of Anxiolytic Effects \\
\hline Psychiatry & $6 / 8$ & Anxiety Disorders & Calming Effect, BAI-S, Subjective Score, BP, HR \\
\hline Psychiatry & $3 / 3$ & PTSD & Calming Effect \\
\hline Pain Management & $6 / 8$ & Situational Anxiety & Calming Effect, Subjective Score \\
\hline Pain Management & $3 / 3$ & Acute Anxiety & Calming Effect \\
\hline Total & $18 / 22$ & & 29 \\
\hline
\end{tabular}

Three of the six responders noted that $\operatorname{Pan} X^{\circledR}$ Atenolol Scopolamine compared favorably to benzodiazepines based upon their prior experiences. Thus, they perceived a benzodiazepine-like calming effect. Another responder noted regarding prior medication use that he had tried "everything in the books and nothing works", yet "this medication has been the only medication that I have noticed a real difference".

Heart rates were determined at the time of drug administration (pre-treatment) and following administration (post-treatment) at 1 hour and at 4-5 hours. The mean heart rates were: pre-treatment $93.6 \mathrm{bpm}$; 1 hour post-treatment $77.0 \mathrm{bpm}$; and 4-5 hours post-treatment $79.7 \mathrm{bpm}$. Thus, there was a reduction in average heart rate of approximately $16 \mathrm{bpm}$ following drug administration. This level of reduction is consistent with the historically reported effects of a beta blocker. Blood pressures were also monitored before and after treatment, and revealed a reduction by $10.1 \mathrm{mmHg}$ systolic and $5.3 \mathrm{mmHg}$ diastolic at 1 hour and an even greater reduction at 4-5 hours. Again, this is consistent with the known effects of a beta blocker.

The side effects noted included dryness of the mouth and mild sedation. It is possible that the mild sedation might have been due to coincident use of other anxiolytic prescription drugs in some or all of the patients affected in this manner.

Case series in psychiatry: Benjamin et al. is a case report of a patient with post-traumatic stress disorder (PTSD) experiencing acute anxiety episodes following psychotherapy sessions [27]; Benjamin and Dooley and Benjamin and Dooley are two additional case reports of patients with PTSD $[28,30]$. All three patients reported a calming effect, with some minor side effects, for the treatment of breakthrough anxiety.

The case reports support the use of compounded Atenolol $25 \mathrm{mg}$ - Scopolamine $\mathrm{HBr} 0.2 \mathrm{mg}$ for acute anxiety, even in tough benzodiazepine-primed patients. The patients experienced acute anxiety episodes and were effectively treated prn. They were seen in either a residential dual diagnosis setting or as an outpatient consult.

The two patients treated in a residential treatment setting had PTSD, along with Major Depressive Disorder and Generalized Anxiety Disorder [27,28]. They had histories of benzodiazepine abuse and had failed Hydroxyzine for acute bouts of anxiety. The patients were on complex multiple medication regimens including SSRIs, an antipsychotic, and Gabapentin. The other patient, who was seen as an outpatient consultation, had diagnoses of PTSD and MDD and was stable on an SNRI and Prazosin [30]. He also had a history of alcohol and Methamphetamine use, and despite trials in the past with benzodiazepines, was interested in a non-addictive option for the treatment of anxiety. Acute anxiety was the common issue to all three. Symptoms included agitation, increased heart rate, shaking (tremors), shortness of breath, and fears of losing control. The patients had been detoxed prior to the physiciansponsored studies.

All described the compounded $\operatorname{Pan} \mathrm{X}^{\circledR}$ medication working within about 20-30 minutes. One patient tried it sublingually with resolution of symptoms within 15 minutes. The perceived calming effects lasted 4-6 hours. Two of the patients compared Pan $X^{\circledR}$ Atenolol - Scopolamine as closely resembling the effects of Klonopin, a benzodiazepine. And, one of these patients noted a reduction in subjective anxiety from 9-10 down to 3 (10 point scale).

None of the patients noted blurred vision or confusion. The side effects noted in at least one patient included dry mouth, some slight imbalance, and mild sedation. As evidenced by multiple trials of the drug combo on separate days by each patient, the benefits were consistent and the side effects were tolerable. 
With one patient blood pressure and heart rate measurements were recorded prior to dosing and at 1 and 4 hours post treatment and for three separate treatments [27]. There was a drop in heart rate at one hour, and this resolved by 4 hours. During one of the three treatment periods the systolic blood pressure dropped $12+\mathrm{mmHg}$ ranges at 1 and 4 hours. Reductions in heart rate (and BP) are presumably due to the beta blocker, which was included in the drug combo to address palpitations and tachycardia during acute anxiety episodes.

Case series in pain management: Thomas et al. is a case series of three patients with acute anxiety symptoms treated prn with compounded Atenolol 25 - Scopolamine $\mathrm{HBr} 0.2$ [29]. Three patients were evaluated in a pain management clinic, and were provided compounded drug for prn use during episodes of acute anxiety [29]. One of the patients was on opioids. All three reported anxiety symptoms including anxiety (anxiousness), palpitations, and shortness of breath. One patient also noted nausea, vomiting, headaches, and numbness and tingling. All three patients reported a calming effect of the medication, with some possible minor and welltolerated side effects. Two of the patients noted symptom resolution after drug administration within 10 to 15 minutes with perceived calming benefits lasting from 2 to 6 hours. One patient reported some dizziness, one reported dry mouth, and another reported some numbness and tingling on the skin of her forearms. All three expressed interest in further use of this compounded medication.

Open label study involving a medical procedure in pain management: Thomas and Dooley (2018) is a study of eight patients experiencing situational anxiety in anticipation of a medical procedure [31]. The patients were assessed in a pain management clinic using a customized questionnaire. Each patient was tested one time. Within 10-20 minutes of drug administration of compounded Atenolol 25 - Scopolamine 0.2, the patients commenced an electromyography (EMG) procedure. The patients were assessed prior to and during the procedure.

Seven of the eight patients reported a perception of a calming effect. Six of the eight patients were deemed as responders to the drug treatment, with an overall subjective anxiety score from an average of 6.3 points prior down to an average of 2.7 points during the procedure (10-point scale). The six responders also reported quantitative reductions in the number and/or severity of individual anxiety symptoms. Eight individual symptoms were assessed: tachycardia, sweating, nausea, numbness, shortness of breath, headache, trembling (tremors), and dry mouth. In at least one of the six responders, each of the eight individual symptoms was reduced or ceased by drug treatment. In three of the six responders all of their multiple symptoms ceased.

Two of the eight patients were deemed as non-responders. None of the eight patients reported experiencing any side effects. All reported being clear minded; none were aware of any impairment of cognition.
It should be noted that the time interval between drug administration and commencing the EMG procedure was very short (10-20 minutes). This parameter is considered to be less than ideal. Had the patients waited a minimum of 30-60 minutes prior to the procedure to facilitate absorption, one may reasonably speculate that the number of responders and/or the magnitude of the response might have been higher in this open label study.

This open label study provides evidence that Atenolol Scopolamine may be used effectively for situational anxiety, and in this case the "trigger" was anticipation of a medical procedure. Four of the 6 responders reported having prescriptions to other anxiolytics, including benzodiazepines.

\section{Summary and Discussion}

The physician-sponsored studies of compounded $\operatorname{Pan} \mathrm{X}^{\circledR}$ Atenolol - Scopolamine in 22 patients [27-32] demonstrated a fast-acting and self-perceived calming effect orally within $<60$ minutes or mucosally (orally-disintegrating) within $<30$ minutes. The self-perceived calming effect was reported by 19 of 22 patients. It persisted for multiple hours, with some patients noting that the self-awareness of a calming effect lasted up to 8 hours. Minor and well tolerated side effects were noted (e.g., dry mouth, mild sedation), and occurred in about half of the patients. None of the side effects were serious or unanticipated with regard to the historic side effect profiles for either Scopolamine or Atenolol as monotherapies. In other words, there was no clinical evidence of any unusual side effect(s) or unforeseen drug-drug interactions.

It should be noted that the selected doses for both active ingredients are considered "low" in this compounded drug combo of Atenolol $25 \mathrm{mg}$ - Scopolamine $\mathrm{HBr} 0.2 \mathrm{mg}$. Atenolol is routinely prescribed for hypertension or other cardiovascular conditions at oral doses of 25-100 mg [7]. Scopolamine is approved over-the-counter (OTC) for motion sickness in Australia, Europe, UK, and Canada at $0.3 \mathrm{mg}$ (or 0.6 mg) [17]. In addition, the selected "low" doses for this particular PanX drug combo have been historically safe for each agent as oral Rx or OTC monotherapies.

Beyond the anxiety studies per se, the cardiovascular effects (i.e., heart rate and blood pressure) of Atenolol $50 \mathrm{mg}$ and/or Scopolamine $\mathrm{HBr} 0.3 \mathrm{mg}$ have been reported elsewhere in 12 healthy "normal" subjects, including during exposure to a sauna to experience a high heat environment [33]. Atenolol 50 $\mathrm{mg}$ (oral) at two hours reduced the mean baseline heart rate by $13 \mathrm{bpm}$. In contrast, Scopolamine $\mathrm{HBr} 0.3 \mathrm{mg}$ (oral) had little-to-no effect (i.e., only $2 \mathrm{bpm}$ reduction and within the Standard Deviation of baseline HR). Furthermore, the heart rate upon co-administration of both oral drugs (57 bpm) mirrored the results of Atenolol alone $(59 \mathrm{bpm}$ and within the Standard Deviation of the co-administration of both drugs). Thus, Scopolamine alone had little-to-no effect and did not counteract or abrogate the cardiovascular suppression effects of Atenolol. This was true while under normal resting conditions or under heat-induced stress. 
The direct comparisons of heart rate and blood pressure between the published cardiovascular study of Atenolol $50 \mathrm{mg}$ with or without Scopolamine $\mathrm{HBr} 0.3 \mathrm{mg}$ [33] and the study in Anxiety Disorders with Atenolol $25 \mathrm{mg}$ - Scopolamine $\mathrm{HBr} 0.2$ mg [32] provide strong evidence of a safe and effective cardiovascular contribution of Atenolol within the PanX ${ }^{\circledR}$ combination, which is coincident with a substantial reduction in anxiety symptoms assessed by the BAI-S and subjective anxiety scores. This strongly suggests a pharmacologic contribution of the beta blocker component upon (at least) the heart rate, blood pressure, and palpitation symptoms in anxious psychiatric patients. Most or all of the other symptoms affected by the combination therapy (e.g., CNS anxiousness) are likely to be due to the Scopolamine component.

At least six of the 22 patients commented that Atenolol 25 $\mathrm{mg}$ - Scopolamine $\mathrm{HBr} 0.2 \mathrm{mg}$ produced anxiolytic effects similar to that of benzodiazepines from their prior experiences $[27,28,30,32]$. The authors also expect that $\operatorname{Pan} X^{\circledR}$ drug combinations will be useful in the treatment of anxiety symptoms in patients affected by Substance Use Disorders (SUDs), including breakthrough anxiety while patients are being ineffectively treated with other medications and/or psychological counseling. As evidence thereof, three of the patients in one of the open label studies were being treated in the residential setting for SUDs, such as alcohol or opioids [32]. Furthermore, those who have abused or are addicted to benzodiazepines are especially at-risk and are in need of anxiolytic alternatives. The $\operatorname{Pan}^{\circledR}{ }^{\circledR}$ approach may serve as a fast-acting prn anxiolytic similar to a benzodiazepine, yet without using active ingredients known to have the potential for dependence, addiction, or abuse.

\section{Conclusion}

Based upon the published results of $18 / 22$ patients that were responders when treated with Atenolol - Scopolamine for various anxiety conditions, the potential medical indications for this (or other) beta blocker - antiemetic antimuscarinic drug combinations include DSM-5 anxiety disorders (e.g., Generalized Anxiety Disorder, Social Anxiety Disorder, Panic Disorder +/- Agoraphobia, Specific Phobias), situational anxiety (e.g., medical and dental procedures), anxiety associated with PTSD, and breakthrough anxiety in SUDs. One of more of these potential indications could be pursued for further clinical development leading to a product approval in the US or elsewhere. Effective prn anxiolytics without using DEA Controlled Substances known to exhibit dependence, abuse, and/or addiction would have great clinical utility in the treatment of more than 45 million adults adversely affected by anxiety disorders in the US and more than 250 million worldwide. Thus, $\operatorname{Pan}^{\circledR}{ }^{\circledR}$ drug combinations may be alternatives to prn benzodiazepines.

\section{Disclosures and Acknowledgements}

The $\operatorname{Pan} \mathrm{X}^{\circledR}$ anxiolytic technology was developed by $\mathrm{Dr}$. Dooley, an employee and shareholder of Trends in Pharma Development LLC (Birmingham, AL). The medications are subject to four issued patents: US 9,446,030, US 9,517,231, US 9,616,052, Canada 2,955,575, and a foreign PCT patent application. Dr. Benjamin is a consultant to and shareholder in TPD LLC. Dr. Thomas has nothing to disclose.

\section{References}

1. Costa e Silva JA (1998) The public health impact of anxiety disorders: a WHO perspective. Acta Psychiatr Scand Suppl 393: 2-5.

2. Kessler RC, Chiu WT, Jin R, Rusico AM, Shear K, et al. (2006) The epidemiology of panic attacks, panic disorder, and agoraphobia in the National Comorbidity Survey Replication. Arch Gen Psychiatry 63: 415-24.

3. Griffiths RR, Weerts EM (1997) Benzodiazepine selfadministration in humans and laboratory animals--implications for problems of long-term use and abuse. Psychopharmacology (Berl) 134: 1-37.

4. Zamorski MA, Albucher RC (2002) What to do when SSRIs fail: eight strategies for optimizing treatment of panic disorder. Am Fam Physician 66: 1477-84.

5. Shelton RC, Harvey DS, Stewart PM, Loosen PT (1993) Alprazolam in panic disorder: a retrospective analysis. Prog Neuropsychopharmacol Biol Psychiatry 17: 423-34.

6. Dooley TP (2015) Treating anxiety with either beta blockers or antiemetic antimuscarinic drugs: A review. Mental Health in Family Medicine 11: 89-99.

7. Lopez-Sendon J, Swedberg K, McMurray J, Tamargo J, Maggioni AP, et al, (2004)Expert consensus document on beta-adrenergic receptor blockers. Eur Heart J 25: 1341-62.

8. Neftel KA, Adler RH, Käppeli L, Rossi M, Dolder M et al. (1982) Stage fright in musicians: a model illustrating the effect of beta blockers. Psychosom Med 44: 461-9.

9. Kraus ML, Gottlieb LD, Horwitz RI, Anscher M (1985) Randomized clinical trial of atenolol in patients with alcohol withdrawal. N Engl J Med 313: 905-909.

10. Horwitz R, Gottlieb LD, Kraus ML (1989) The efficacy of atenolol in the outpatient management of the alcohol withdrawal syndrome. Results of a randomized clinical trial. Arch Intern Med 149: 1089-93.

11. Gottlieb LD, Horwitz RI, Kraus ML, Segal SR, Viscoli CM (1994) Randomized controlled trial in alcohol relapse prevention: role of atenolol, alcohol craving, and treatment adherence. J Subst Abuse Treat 11: 253-258.

12. Ekeberg O, Kjeldsen SE, Greenwood DT, Enger E (1990) Effects of selective beta-adrenoceptor blockade on anxiety associated with flight phobia. J Psychopharmacol 4: 35-41.

13. Liebowitz MR, Schneier F, Campeas R, Hollander E, Hatterer J, et al. (1992) Phenelzine vs atenolol in social phobia. A placebocontrolled comparison. Arch Gen Psychiatry 49: 290-300.

14. Gupta D, Srivastava S, Dubey RK, Prakash PS, Singh PK, et al. (2011) Comparative evaluation of atenolol and clonidine premedication on cardiovascular response to nasal speculum insertion during trans-sphenoid surgery for resection of pituitary adenoma: A prospective, randomised, double-blind, controlled study. Indian J Anaesth 552: 135-140. 
15. Ellis ME, Sahay JN, Chatterjee SS, Cruickshank JM, Ellis SH (1981) Cardioselectivity of atenolol in asthmatic patients. Eur J Clin Pharmacol 21: 173-176.

16. Navas EV, Taylor DO (2010) Can patients with COPD or asthma take a beta-blocker? Cleve Clin J Med 77: 498-499.

17. Corallo CE, Whitfield A, Wu A (2009) Anticholinergic syndrome following an unintentional overdose of scopolamine. Ther Clin Risk Manag 5: 719-23.

18. Imai K, Ikenaga M, Kodama T, Kanemura S, Tamura K, (2013) et al, Sublingually administered scopolamine for nausea in terminally ill cancer patients. Support Care Cancer 21: 2777-81.

19. Gray MY (2007) The use of anticholinergics for the management of terminal secretions. Evidence Matters (Hospice Pharmacia newsletter) 1: 1-6.

20. Khajavi, D, Farokhnia M, Modabbernia A, Ashrafi M, Abbasi SH, et al. (2012) Oral scopolamine augmentation in moderate to severe major depressive disorder: a randomized, double-blind, placebo-controlled study. J Clin Psychiatry 73: 1428-33.

21. Houde A (1906) Scopolamine: A Physiological and Clinical Study. Am J Clin Med 13: 365-367.

22. Drevets WC, Furey ML (2010) Replication of scopolamine's antidepressant efficacy in major depressive disorder: a randomized, placebo-controlled clinical trial. Biol Psychiatry 67: 432-8.

23. Furey ML, Khanna A, Hoffman EM, Drevets WC (2010) Scopolamine produces larger antidepressant and antianxiety effects in women than in men. Neuropsychopharmacology 35: 2479-88.

24. Furey ML, Drevets WC (2006) Antidepressant efficacy of the antimuscarinic drug scopolamine: a randomized, placebocontrolled clinical trial. Arch Gen Psychiatry 63: 1121-9.
25. Wang JC, Hinrichs AL, Stock H, Budde J, Allen R, et al, (2004) Evidence of common and specific genetic effects: association of the muscarinic acetylcholine receptor M2 (CHRM2) gene with alcohol dependence and major depressive syndrome. Hum Mol Genet 13: 1903-11.

26. Witkin JM, Overshiner C, Li X, Catlow JT, Wishart GN, et al. (2014) M1 and $\mathrm{m} 2$ muscarinic receptor subtypes regulate antidepressant-like effects of the rapidly acting antidepressant scopolamine. J Pharmacol Exp Ther 351: 448-56.

27. Benjamin A, Mollner A, Dooley TP (2017) Atenolol - Scopolamine Combination Drug Decreases Acute Anxiety Post Trauma Therapy Session. Ann Depression Anxiety 4: 1086.

28. Benjamin AB, Dooley TP (2017) A compounded, nonbenzodiazepine option for treating acute anxiety. Current Psychiatry 16: e1-e2.

29. Thomas T, Benjamin AB, Dooley TP (2017) Treatment of Acute Anxiety Episodes in Patients Using a Fast-Acting Beta Blocker Scopolamine Combination Drug. Ann Depression Anxiety 4: 1088.

30. Benjamin AB, Dooley TP, (2017) Beta Blocker-Scopolamine Combination Drug Decreases Acute Anxiety in a PTSD Patient - A Case Report. ARC J Psychiatry 2: 1-4.

31. Thomas Ty, Dooley TP (2018) Treatment of Anxiety Prior to a Medical Procedure using an Atenolol - Scopolamine Combination Drug. J Depression Anxiety 7: 303.

32. Benjamin AB, Dooley TP (2019) Anxiolytic Benefits of Compounded Atenolol - Scopolamine in Eight Patients in Psychiatry. Personalized Medicine in Psychiatry.

33. Kukkonen-Harjula K, Oja P, Vuori I, Pasanen $\mathrm{M}$, Lange $\mathrm{K}$, et al. (1994) Cardiovascular effects of Atenolol, scopolamine and their combination on healthy men in Finnish sauna baths. Eur J Appl Physiol Occup Physiol 69: 10-15. 